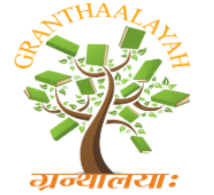

INTERNATIONAL JOURNAL OF RESEARCH GRANTHAALAYAH A knowledge Repository

RACSIT - 17

\title{
WEARABLE REAL TIME HEALTH TRACKERS AND ASSISTED- MOBILITY FOR NON-AMBULANT PATIENTS
}

\author{
Vidya Shree $\mathbf{G}^{1}$, Sharanabasappa ${ }^{2}$ \\ ${ }^{* 1,2}$ Department of ECE, Don Bosco Institute of Technology, Bangalore, India
}

DOI: https://doi.org/10.29121/granthaalayah.v5.i4RACSIT.2017.3349

\begin{abstract}
This paper presents an approach to facilitate mobility for the non-ambulant patients using eye movements and wearable health tracking. The patient's wheelchair movement is controlled using eye ball movements and also some biomedical assistance functionalities are considered to match the current day needs. The proposed work first detects face from input video, then eye portion will be localized, and finally eye ball (pupil) is detected and tracked using computer vision techniques. The direction of movement is assessed and a command is disseminated to the wheelchair control system. The wearable sensors and lor alarms mounted on patients will update current health status indications on to the monitoring panels. The wearable devices have evolved very smartly which are capable enough to take care of the patient health in real time even during assisted mobility.
\end{abstract}

Keywords: Computer Vision; Image Processing Techniques; Hough Transform; Wheelchair Control; Real-Time Application.

Cite This Article: Vidya Shree G, and Sharanabasappa. (2017). "WEARABLE REAL TIME HEALTH TRACKERS AND ASSISTED-MOBILITY FOR NON-AMBULANT PATIENTS." International Journal of Research - Granthaalayah, 5(4) RACSIT, 41-46. https://doi.org/10.29121/granthaalayah.v5.i4RACSIT.2017.3349.

\section{Introduction}

Although wheelchairs provide a means for independent movement from one place to another, turning the wheels by own hands is a challenge if the person possesses limited movement capability. Driving and control of wheelchair by hand poses a great impediment for a physically challenged person with limited physical ability but a real time eye-gaze tracking based control system can go a long way in easing such cases. In such cases, a person can move their eyes which can be used to transfer instructions towards intended direction of movement. Accurate real-time detection of the eye gazing can assist them to control the movement of wheelchairs or other movable/flexible instruments, such as the flexible part of the bed or a trolley (for bed 
ridden patients). The idea here is to track the pupil movement and interpret the images for further control of devices.

The medical-grade monitor lets people monitor their heart health, Qardio's proprietary sensor technology that records more than 20 million data points that stream to a companion smart phone app. The data can be shared with medical professionals. The strap is worn around the chest and records continuous ECG, heart rate, heart rate variability, respiratory rate, temperature and activity data, and the strap can also work alongside Qardio's other products including their smart blood pressure monitor, a wireless scale and a the QardioMD dashboard for doctors.

Valence cell announced the launch of two new versions of its Benchmark sensor systems - one system specifically for hearables (the new version is smaller to allow for faster time to market) and one for wearables worn on the wrist and arm. Both new modules measure continuous heart rate, VO2 and VO2 max, resting heart rate, caloric burn and recovery. Philips showed off its suite of connected health tools - The Avent uGrow digital parenting platform, which is a companion app paried with Philips' smart baby monitor and ear thermometer; Philips Dream Station Go portable CPAP device, which is designed for users to take anywhere; the connected Sonic are toothbrush and the Philips Heart Health program, which is an app-based behavior change program that pairs the Philips heath watch with mobile coaching. Philips also announced a partnership with Daimler for the Mercedes Benz Fit \& Healthy project to provide drivers with health information while driving.

\section{Related Work}

Circular Hough Transform (CHT) is a one of the benefit algorithm to detect pupil region. [19], [19] used CHT to detect eye and pupil. However none of them could detect eye gaze using CHT, because CHT well works only on circular objects. If the eye is not forward gazing, the pupil is no longer circular so CHT fails to detect them if the condition of objects edges being perfectly circular is not related.

In [19], the perfect circular condition is relaxed then CHT starts to detect lots of object of objects as circular even though they are not for this reason CHT is not usually used in eye gazing tracking. Even though they detect the pupil position only if samples were almost forward gazing and they use dark pupil center to detect the pupil center which is not possible in real time because there will be light reflection in pupil. For this reason we are using image processing technique to avoid multiple object detection even if edge condition is relaxed.

\section{Proposed System}

If the eye is not forward gazing, the pupil is no longer circular. So, CHT fails to detect them if the condition of object edge's being perfectly circular is not relaxed. If the perfect circular condition is relaxed, then CHT starts to detect a lot of objects as circular even though they are not. For this reason CHT is not usually used in Eye gaze tracking. The computer vision image processing technique is used to detect the eyes and the pupils. Any one eye pupil is tracked to assess the directions of movements. The computer vision tool box uses HOG features and HAAR classifier for the detection of the eyes and the pupils. The proposed system for non-ambulant 

architecture. The proposed work flow is given in figure 1.

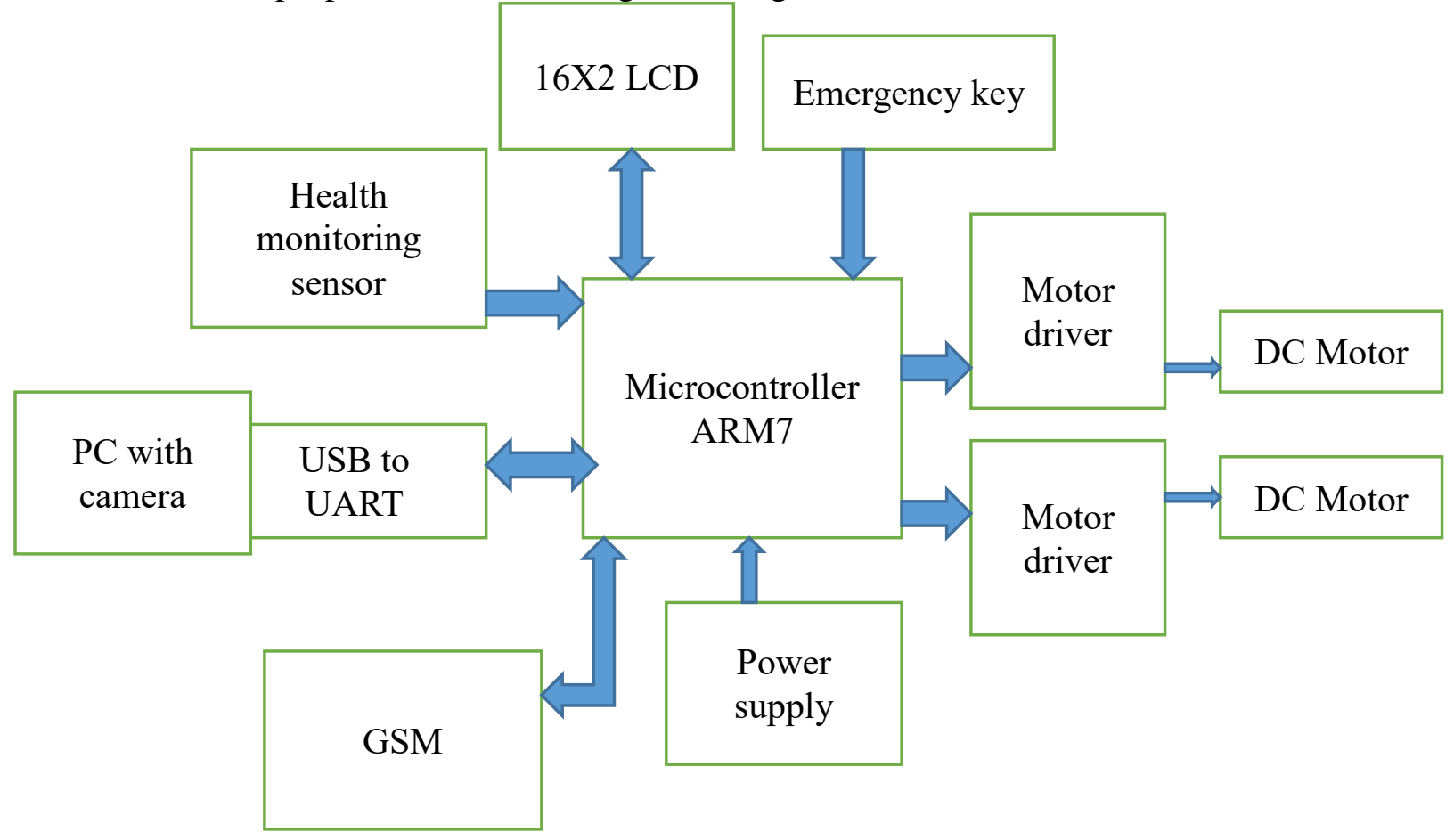

Figure 1: Proposed system Architecture

\subsection{Embedded Unit}

ARM7 microcontroller is heart of the project where all data processing monitoring and controlling can be done through this. LCD display is used for see the status of project and all current action on screen. Basically ultrasonic sensor is used for monitoring the obstacle in the path and giving signal to the microcontroller. The main aim of project is movement of wheel chair by taking the input from eye ball of patient based on his eye ball rotation the movement or direction of chair is controlled. This task is achieved in our project by the help ZIBEE and MATLAB image processing. ZIGBEE is wireless data communication device for interface between microcontroller and PC. Here emergency key is used for helping the patient for his needs and concern of safety, if he I want help of parent or friend that time he/she can press this key using GSM technology will send the SMS alert to them and location also tracked. For running our wheel chair DC motors are used with the help of motor drivers.

1) Eye Pupil tracking using computer vision image processing technique.

2) Wheelchair control using eye pupil tracking commands.

3) Patient Health Monitoring using wearable health trackers

\subsection{Computer Vision Image Processing Technique}

This detects the eyes and the pupils. Any one eye pupil is tracked to assess the directions of movements. The HOG features and HAAR classifier are used for the detection and tracking of eye pupils. 
The wearable sensors data taken from the embedded module are used to monitor the health of the patient. The abnormal conditions are monitored and are indicated to the respective medical or para-medical guardians.

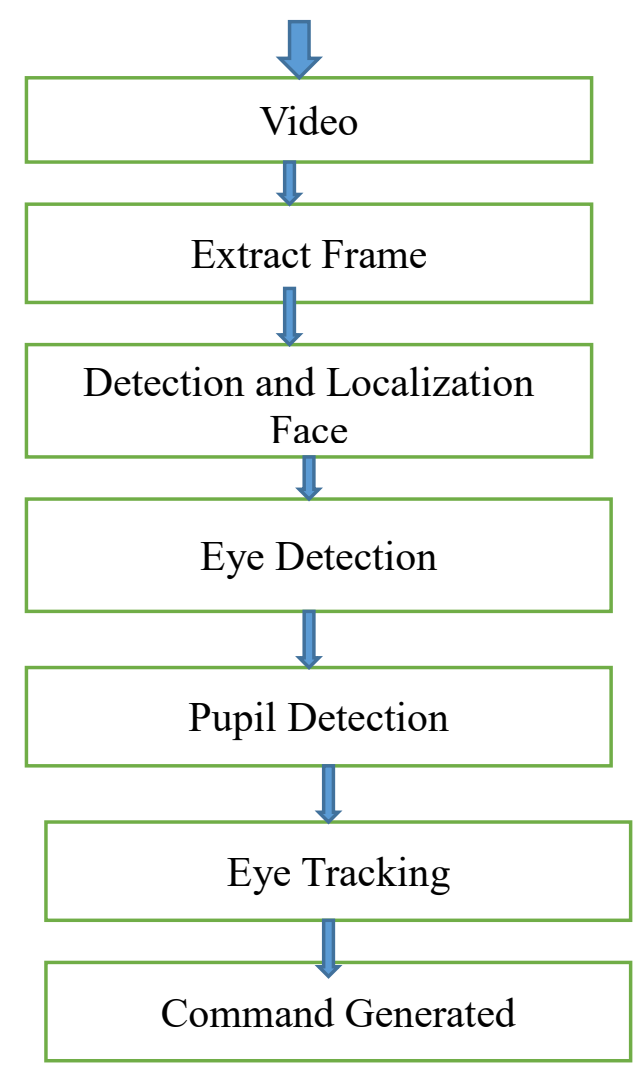

Figure 2: Image processing module

\subsection{Image Acquisition}

To keep the method cost-effective and to simulate worst real-time conditions, no high-resolution cameras were used. Rather a cell phone camera with 5 MP resolutions, 720p@30fps was used. The cell phone was situated at a certain position of the wheel chair focusing on one eye of the user. Continuous video is captured and the video data is sent to the PC for processing. Then image frames are extracted from the continuous video data at certain small time interval. The acquired frames are then used for subsequent processing.

\subsection{Eye-gaze Tracking}

Considering the processing requirements and memory and speed constraints, we take the image of a single eye of that person. The camera is focused in such a way that only one eye can be captured in the image frame. The samples of such images are presented in Fig. 2. The eye gazing can be in any of the left, right, upward or forward direction. The closed eye looks similar to the downward gazing eye in the image. So, we set the control of the vehicle as the following- left gazing eye means the person wants to move left ward, right gazing eye means the person wants to move right ward, upward gazing eye means the person wants to move forward, downward 
gazing eye or closed eye means the person wants to move backward and forward gazing eye means the person wants to be in the the same place as s/he is now. The first step of the image processing part is image resizing.

\section{Experiments and Discussions}

The proposed system has been developed and experimented with a prototype of wheelchair. The camera was set about $50 \mathrm{~cm}$ apart from the eye. In order to capture image of only one eye, the camera zoom capability used is 4 X.the camera was at about height from the handle of the chair. The height of camera can be adjusted according to the height of the person .various controls to wheelchair were given using eye tracking of multiple users to determine the accuracy of proposed approach and the result the proposed algorithm provides $81.2 \%$ accurate estimation on average which is satisfactory considering it is computationally lighter and so suitable for intended real-time applications. The accuracy could be more if we could use real disabled persons.

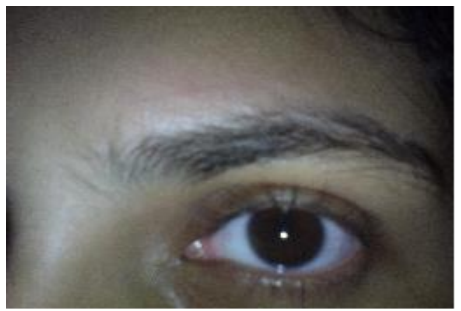

(a)

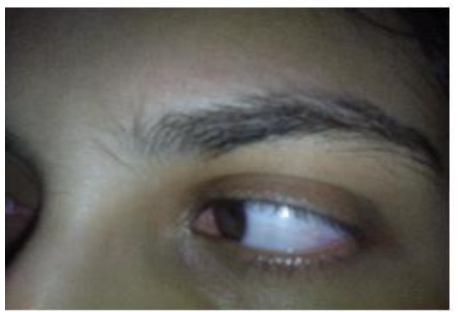

(d)

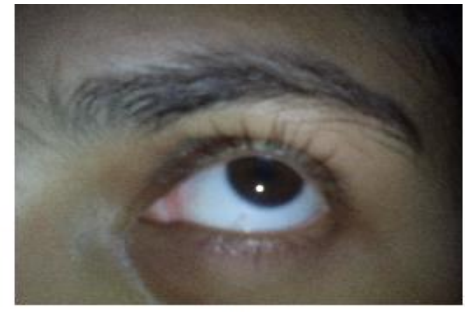

(b)

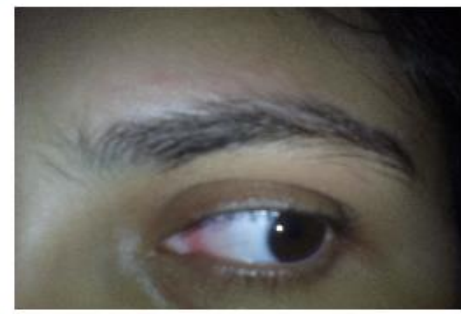

(e)

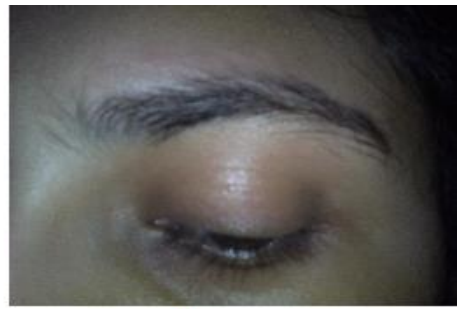

(c)

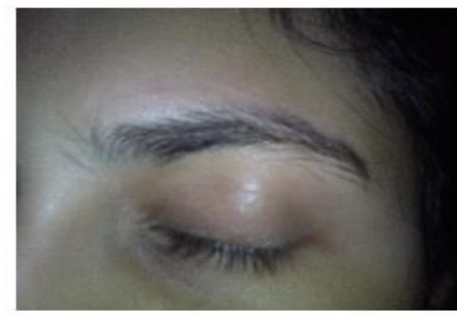

(f)

Figure 3: Sample image of one eye, (a) forward gaze, (b) upward gaze, (c) Downward gaze, (d) rightward gaze, (e) leftward gaze, (f) closed eye.

\section{Conclusions}

This paper presents Real-time computational efficient approach for wheelchair direction control using limited eye movement tracking system using computer vision technique to help physically challenged people who suffered from driving the wheel chair by own. There is also emergency key provided which when pressed will trigger an alarm as well as send the message and we also used two sensors two check patient health in real time. The proposed system can be used for other humanitarian applications as well such as control of sophisticated games

\section{References}

[1] S. K. Card, A. Newell, and T. P. Moran, “The psychology of human computer interaction,”, 1983. 
[2] B. Shackel, "Note on mobile eye viewpoint recording," JOSA, vol. 50, no. 8, pp. 763-768, 1960.

[3] C. H. Morimoto, and M. R. M. Mimica, "Eye gaze tracking techniques for interactive applications," Computer Vision and Image Understanding, vol. 98, no. 1, pp. 424, 2005.

[4] A. Duchowski, Eye tracking methodology: Theory and practice, volume 373. Springer Science \& Business Media, 2007.

[5] T. Levola, Eye-gaze tracking, July 6 2004. US Patent 6,758,563.

[6] D. W. Hansen, Eye gaze tracking, July 8 2008. US Patent App. 13/003,030.

[7] B. L. Nguyen, "Eye gaze tracking," In Computing and Communication Technologies, 2009. RIVF09. International Conference on, pp. 1-4. IEEE, 2009.

[8] C. Ware, and H. H. Mikaelian, "An evaluation of an eye tracker as a device for computer input2," In ACM SIGCHI Bulletin, vol. 17, pp. 183- 188, 1987.

[9] J. L. Levine, An eye-controlled computer, IBM Research Division, TJ Watson Research Center, 1981.

[10] R. Stiefelhagen, J. Yang, and A. Waibel, "Tracking eyes and monitoring eye gaze," In Proc. Workshop on Perceptual User Interfaces, pp. 98-100, 1997.

[11] Hansen, D.W. and Pece, A.E., "Eye tracking in the wild." Computer Vision and Image Understanding, vol. 98, no. 1, pp. 155-181, 2005.

[12] Xia Liu -Real-time eye detection and tracking for driver observation under various light conditionsl,Intelligent Vehicle Symposium, June 2002.

[13] Zhang Guang Yuan -A real-time eye detection system based on the active IR illumination\| Control and Decision Conference. 2008.

[14] M. Eriksson, and N.P. Papanikotopoulos, -Eye-tracking for detection of driver fatigue, „l in Proc. Int. Conf. Intelligent Transportation Systems, Boston, pp. 314- 319, 1997.

[15] S. Singh, and N.P. Papanikolopoulos, -Monitoring driver fatigue using facial analysis techniques, $\|$ in Proc. Int. Conf. Intelligent Transportation Systems, Tokyo, Japan, pp. 314-318, 1999.

[16] Paul Viola and Michael Jones "Rapid Object Detection using a Boosted Cascade of Simple Features", Conference on computer vision and pattern recognition, 2001.

[17] M. Turk, and A. Pentland, "Eigen faces for Recognition", Journal of Cognitive Neuro sicence, Vol. 3, No. 1, 1991

[18] R. Grace, -Drowsy driver monitor and warning system,\| in Proc. Int.Driving Symp. Human Factors in Driver Assessment, Training andVehicle Design, 2001.

[19] Khairosfaizal, W. W. M. and Nor'aini, A. J, "Eyes detection in facial images using circular hough transform." In Signal Processing \& Its Applications, 2009. CSPA 2009. 5th International Colloquium on, pp. 238-242. IEEE, 2009.

[20] M. Soltany, S. T. Zadeh, and H. R. Pourreza, "Fast and accurate pupil positioning algorithm using circular Hough transform and gray projection." In International Conference on Computer Communication and Management (CSIT), vol. 5, pp. 556-561. 2011.

*Corresponding author.

E-mail address: Vidyagowda24@gmail.com 